IOSR Journal of Engineering (IOSRJEN)

e-ISSN: 2250-3021, p-ISSN: 2278-8719

Vol. 3, Issue 9 (September. 2013), ||V2|| PP 57-64

\title{
Quality-Based Sampling Test Design for Head Gimbal Assembly
}

\author{
Visvas Lerswanichkul, Napassavong Rojanarowan \\ ${ }^{12}$ Department of Industrial Engineering, Faculty of Engineering, Chulalongkorn University, Bangkok, Thailand.
}

\begin{abstract}
This paper has the objective to present the method to reduce the testing activities while maintaining quality. The new electronic sampling test of the Head Gimbal Assembly (HGA) is proposed to replace the current $100 \%$ testing method. The proposed sampling test uses the Discriminant Analysis (DA) technique to predict the testing result of the on-disc test for each HGA based on the variables from previous processes. If the prediction result shows "pass", the company should skip the testing of that HGA but if the prediction result shows "fail", the company should test that HGA as usual. It was found that the proposed sampling test could predict the results with $90.68 \%$ accuracy. If applying this new testing plan in the production, $90.83 \%$ of the HGAs could skip the on-disc test. As a result, the tester usage could be saved up to $10 \%$.
\end{abstract}

Keywords: - Sampling Testing, Prediction, Discriminant Analysis, Head Gimbal Assembly, Hard Disk Drive

\section{INTRODUCTION}

The hard disk drive industry is one of the most important electronic industries in the world. Nowadays, hard disk becomes a part of daily life of people. The hard disk drive (HDD) is used for storing data in a lot of electronic devices such as computers, laptops, digital cameras and others. Competition in the hard disk drive industry is highly competitive. Manufacturers need to continue to improve the product quality as well as develop manufacturing processes to reduce costs and enhance competitiveness. Quality and confidence of customers are important. Thus, quality testing is an important activity of producers for making sure that the devices which will be sent to the customers will be able to meet the customer expectations. However, more quality testing comes with higher cost of testing. Thus, manufacturers in the electronic industry have to find ways to reduce the production costs while maintaining the quality of work delivered to the customers.

\section{PROBLEM DESCRIPTION}

The study of the case study factory found that the ability of the manufacturing processes is not enough to support the demand that is increasing. It was found that the bottleneck process is the electrical testing in the Head Gimbal Assembly process since the number of the electrical testing machines is limited. Product A is a main product of the case study factory. It is used in a desktop computer. The case study factory needs to test 500,000 HGAs of this product per day. The product A uses up 50\% of the capacity of all testing machines in this factory. That means the factory needs to use 60 testing machines for this product. Increasing the capacity of the factory can be done by purchasing more electrical testing machines but it is very expensive. Moreover, the factory has limited work area to install the new testing machines and the factory expansion is too costly. Thus, the case study factory has to find a way to increase the capacity that does not require more investment. This research thus proposes a method to reduce the testing while maintaining the quality of the products without requiring more investment. Next, the manufacturing and testing processes of hard disk drives are described.

\subsection{Hard Disk Drive Manufacturing Processes}

The hard disk drive manufacturing processes can be separated into four main processes as follows:

1) Wafer production - In this process read-write heads are produced in a big lot size in one plate of wafer. One wafer has read-write heads of more than 80,000 heads. Then, the read-write heads are tested by an electrical test before they are sent to the next process.

2) Slider production - In this process a wafer is cut into a read-write head form called slider. The sliders are then tested by an electrical test before they are sent to the next process.

3) Head Gimbal Assembly (HGA) process - This process receives the sliders from the slider production process. Then, the sliders are attached onto the arms called Trace Gimbal Assemblies (TGA) to form the Head Gimbal Assemblies. The HGAs are then tested by an electrical test before they are sent to the next process.

4) Hard Disk Drive (HDD) assembly process. This is the final step of the hard disk drive production. In this process various components such as the HGA, the coil magnetic and the PCBA are combined into a hard disk drive. After the assembly is completed, the HDD is tested by an electrical test and a mechanical test in order to test whether it meets the specific requirements of each product. Fig. 1 shows the HDD manufacturing processes. 


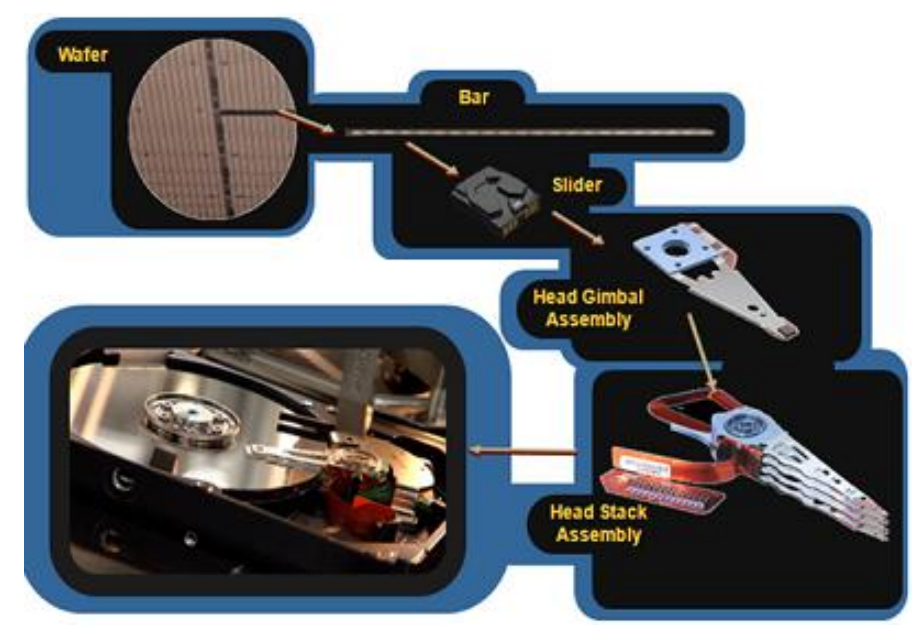

Fig. 1 Hard disk drive manufacturing processes

\subsection{Head Gimbal Assembly Testing Process}

This research focuses on the study of the testing plan in the Head Gimbal Assembly process since it is the bottleneck process. When an HGA finishes the assembly, it is tested by an electrical testing machine. The testing machine performs two tests, which are the off-disk test and the on-disc tests respectively. Before improvement, all HGAs have to enter into both tests. The HGAs after testing can be classified into three groups based on the testing results of both tests as follows.

1) Good HGAs - HGAs in this group can pass both the off-disk and the on-disc test. The Good HGAs are then sent to the HDD assembly process. For product A, $98 \%$ of the HGAs are in this group.

2) Poor HGAs - HGAs in this group can pass the off-disc test but they fail the on-disc test. These HGAs are then scrapped at the HGA process. Actually Poor HGAs are the HGAs that can be operated on the hard disk drives but their read and write ability are poorer than the Good HGAs. For product A, 1.6\% of the HGAs are in this group.

3. Dead HGAs - HGAs in this group fail both the off-disc test and the on-disc test. These HGAs are then scrapped at the HGA process. This group of HGAs cannot operate on hard disk drives. For product A, $0.4 \%$ of the HGAs are in this group.

Before improvement only the HGAs in the group of "Good HGAs" are sent to the hard disk drive assembly process. The Poor HGAs and the Dead HGAs are scrapped at the HGA process. In the hard disk drive assembly process, the Good HGAs can pass the test at the HDD assembly process up to $90 \%$. If hard disk drive assembly process tries to assemble the Poor HGAs into hard disk drives, $60 \%$ of the Poor HGAs can pass the test in the HDD assembly process. Regarding the Dead HGAs, if the HDD process tries to use the Dead HGAs, no HGAs in this group can pass the test at the HDD process.

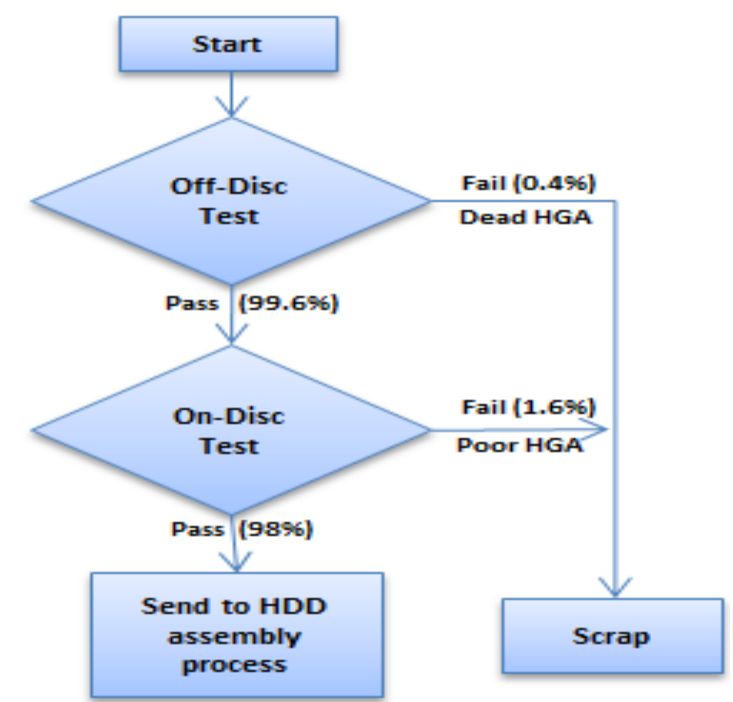

Fig. 2 Process flow of the electrical test at the HGA process before improvement 


\section{PROPOSED HGA ELECTRICAL TESTING PLAN}

Since there is a high percentage of the HGAs that can pass the on-disc test and there is also $60 \%$ of the poor HGAs that can pass the HDD testing process. Thus, the research proposed that instead of testing all HGAs on the on-disc test, some of them should be tested while the others can be skipped. This is called "Sampling Test". The off-disc test still has to be performed for all HGAs since the off-disc test is used to test the very critical properties of the HGAs. The off-disc test and the on-disc test use the same testing machine. Testing only some HGAs in the on-disc test helps reduce the number of testing machines needed and the increased capacity can be used for other products. To be able to skip the on-disc test, it is necessary to have a prediction of the result of the on-disc test and this prediction result should be sufficiently accurate. It was proposed that the data from previous production processes that are the testing results at the Wafer production, the Slider production, and the off-disc test should be used in the prediction of the on-disc test results. If the prediction equation forecasts that the HGA will "pass" the on-disc testing, that HGA can skip the on-disc testing and it will be sent to the next operation but if the prediction equation forecasts that the HGA will "fail" the on-disc test, that HGA needs to be tested by the on-disc test. Fig 3 . shows the process flow of the proposed sampling test at the HGA process.

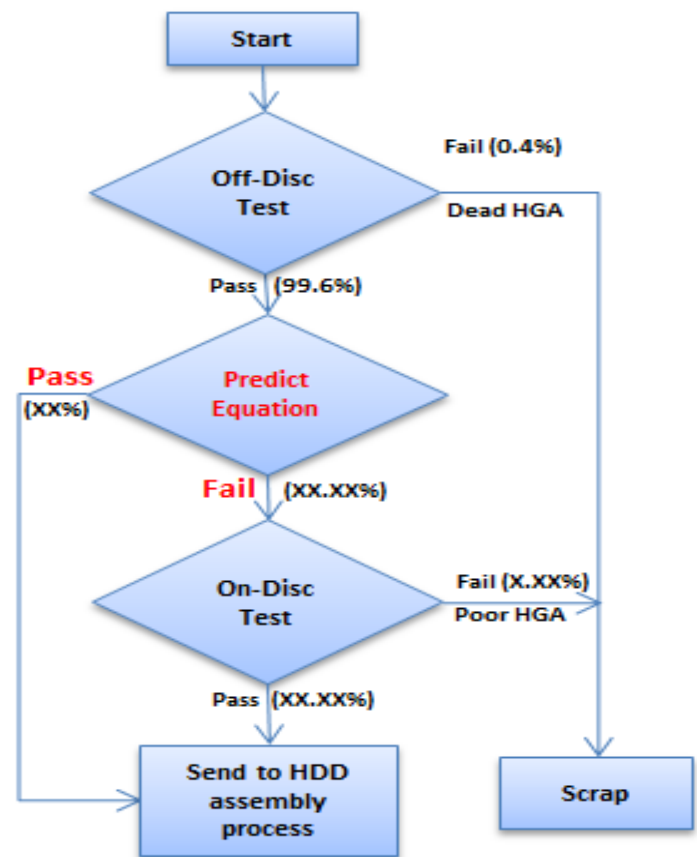

Fig. 3 Process flow of the proposed electrical testing plan (Sampling Test) at the HGA process

Normally, the prediction result can be correct or incorrect. There are four groups of results that can be found as shown in Table 1.

Group 1 - The prediction is "pass" and the actual result is "pass". The HGAs in this group will skip the on-disc test. The testing capacity will be increased and there will be no change in the effect on the quality of the HGAs passed to the HDD assembly process.

Group 2 - The prediction is "pass" and the actual result is "fail". The HGAs in this group will skip the on-disc test. The testing capacity will be increased. The Poor HGAs can pass to the HDD assembly process since the prediction is wrong. Thus these HGAs will adversely affect the yield of the HDD assembly process. Based on Statistics term, this type of error of the prediction is called Type II Error, which means falsely accepting bad parts.

Group 3 - The prediction is "fail" and the actual result is "pass". The HGAs in this group will be tested by the on-disc test. After the HGAs in this group are tested, these HGAs can pass the test. Thus, the HGA assembly process losses the opportunity to skip the test. Regarding the effect on the HDD assembly process, there will be no change in the effect on the quality of HGAs passed to the HDD assembly process. Based on Statistics term, this type of error of the prediction is called Type I Error, which means falsely rejecting good parts.

Group 4 - the prediction is "fail" and the actual result is "fail". The HGAs in this group will be tested by the ondisc test and they will fail the test. Thus, there will be no change in the effect on the yield of the HDD assembly process since the failed HGAs will not be passed to the HDD process. Table 1 summarizes the effect of the proposed sampling test on the HGA and HDD processes. 
Table 1 Effect of the Proposed Sampling Test on the HGA and HDD Processes

\begin{tabular}{|c|c|c|c|c|c|}
\hline $\begin{array}{c}\text { Gro } \\
\text { up }\end{array}$ & $\begin{array}{c}\text { Predicti } \\
\text { on } \\
\text { Result }\end{array}$ & $\begin{array}{c}\text { Actual } \\
\text { Status }\end{array}$ & Action & $\begin{array}{c}\text { Effect on Head Gimbal } \\
\text { Assembly Process }\end{array}$ & $\begin{array}{c}\text { Effect on Hard disk } \\
\text { Drive Assembly } \\
\text { Process }\end{array}$ \\
\hline 1 & Pass & Pass & Skip on-disc test & Increase testing capacity & $\begin{array}{c}\text { No change in effect on } \\
\text { quality }\end{array}$ \\
\hline 2 & Pass & Fail & Skip on-disc test & Increase testing capacity & $\begin{array}{c}\text { Yield loss due to } \\
\text { Poor HGAs }\end{array}$ \\
\hline 3 & Fail & Pass & Perform on-disc test & $\begin{array}{c}\text { Loss of opportunity to } \\
\text { skip the test }\end{array}$ & $\begin{array}{c}\text { No change in effect on } \\
\text { quality }\end{array}$ \\
\hline 4 & Fail & Fail & Perform on-disc test & No Effect & $\begin{array}{c}\text { No change in effect on } \\
\text { quality }\end{array}$ \\
\hline
\end{tabular}

\section{METHODOGY}

This research proposed to use the Discriminant Analysis (DA) as a tool to make the prediction. The DA technique is used to classify objects into groups based on predicting variables, which are of variable type. There have been many researches that used the DA technique to classify objects. For example, the DA was used to classify the mental illness of the Thai Muslim Schizophrentic Patients [1]. It could predict the results with 97.3\% accuracy. The DA was also used in the medical field. It was used to do the classification of antibiotic resistance patterns of indicator bacteria [2]. The DA could classify the object with $63 \%$ accuracy. Moreover, some research compared the performance of DA with other prediction tools such as Case-Based Forecasting and Neural Network. For example, research on bankruptcy prediction [3] found that the Case-Based Forecasting and the Discriminant Analysis had the same performance in classifying groups while the Neural Network had lower accuracy than the two mentioned tools. This research found that the number of variables used in the prediction did not affect the accuracy significantly as shown by the result that the number of predicting variables of 9 and 16 provided the higher accuracy than other number of variables.

\subsection{Data Collection}

The researcher used the tested data collected from the Wafer production process, the Slider production process and the off-disc test as predicting variables. All HGAs were tested on these 12 variables. These variables are shown in Table 2. The data also contained the actual result of the on-disc test. The whole data set were classified into two groups. The first group was called "Analysis Sample", which was used for creating discriminant function. The second group was called "Validation Sample", which was used for validating the accuracy of the results predicted by the discriminate function created from the Analysis Sample.

\subsection{Assumption Checking}

In order to confirm that the data collected were appropriate for being analyzed with the DA technique, the assumptions of the DA had to be validated. The assumptions are related to the following topics: sample size, normal distribution, homogeneity of variances and covariances, and non-multicollinearity.

\subsection{Discriminant Function Creation}

After validating the assumptions using the whole set of data, the discriminant function was created using the Analysis Sample using the method of DA. Then, the Wilk's lambda test was used to test whether the discriminant function was significant in classifying the objects [4]. This research studied both linear discriminant analysis and the quadratic discriminant analysis.

\subsection{Consideration on the Performance of the Prediction}

The performance of the prediction needed to be evaluated using the Validation Sample. The discriminant function was appropriate to be used if the percentage of correct prediction was acceptably high and the probability of Type II error $(\beta)$ was small enough. The value of $\beta$ contributed to the reduction of the yield in the HDD process. Thus, the less $\beta$ was preferred.

\subsection{Decision Making}

The proposed sampling test should be used instead of the current testing procedure if the accuracy of the prediction were acceptable. It was determined that the percentage of the accuracy should be at least $90 \%$ and the $\beta$ value has to be less than $10 \%$. 


\subsection{Data collection}

\section{RESULTS}

The researcher collected a set of data from product A. This data set contained the data of 229,806 HGAs, which was $45.96 \%$ of the number of HGAs tested in one day. There were 225,267 Good HGAs or 98.02 $\%$ of the data, 3,524 Poor HGAs or $1.53 \%$ of the data, and 1,015 Dead HGAs or $0.44 \%$ of the data. The data set contained of the results of the electrical tests based on 1 variable from the Wafer production process, 8 variables from the Slider production process, and 3 variables from the off-disc test as shown in Table 2. Then, the data were separated into two data sets: Analysis Sample and the Validation Sample by half.

Table 2 Variables Used in the DA prediction

\begin{tabular}{|c|l|c|l|}
\hline Number & Testing at Operation & Abbreviation & \multicolumn{1}{|c|}{ Variable Name } \\
\hline 1 & Wafer production & T1 & Writer Electronic Width \\
\hline 2 & Slider production & A1 & Flatness of Writer \\
\hline 3 & Slider production & I1 & Voltage \\
\hline 4 & Slider production & I2 & Signal Symmetry \\
\hline 5 & Slider production & I3 & Resistance \\
\hline 6 & Slider production & I4 & Read Ability \\
\hline 7 & Slider production & I5 & Noise 1 \\
\hline 8 & Slider production & I6 & Noise 2 \\
\hline 9 & Slider production & I7 & Noise 3 \\
\hline 10 & Off-disc test & E1 & Resistance 1 \\
\hline 11 & Off-disc test & E2 & Resistance 2 \\
\hline 12 & Off-disc test & E3 & Resistance 3 \\
\hline
\end{tabular}

\subsection{Assumption Checking}

\subsubsection{Sample size}

Normally the sample sizes of the two groups (pass and fail) should be approximately equal. Regarding our data, the sample size of "pass" group was 225,267 while the sample size of "fail" group was 3,524. The sample sizes of the two groups were significantly different. Poulsen \& French (2004) suggested that unequal sample sizes are acceptable if the sample size of the smallest group exceeds the number of predictor variables or at least 20 [4]. Our small group of data has 3,524 HGAs, which correspond to this assumption.

\subsubsection{Normal distribution}

The data should be used normally distributed to be appropriate to use DA. However, Tabachnick \& Fidell (1996) stated that the violation of the normality assumption is not "fatal" and the results are still reliable as long as non-normality is caused by skewness and not by outliers [5]. All of the variables were not normally distributed even though they had bell shapes. The data based on some variables were skewed with small proportion of outliers as shown in Fig. 4. Thus, in generally the data set satisfied the assumptions of the DA technique.
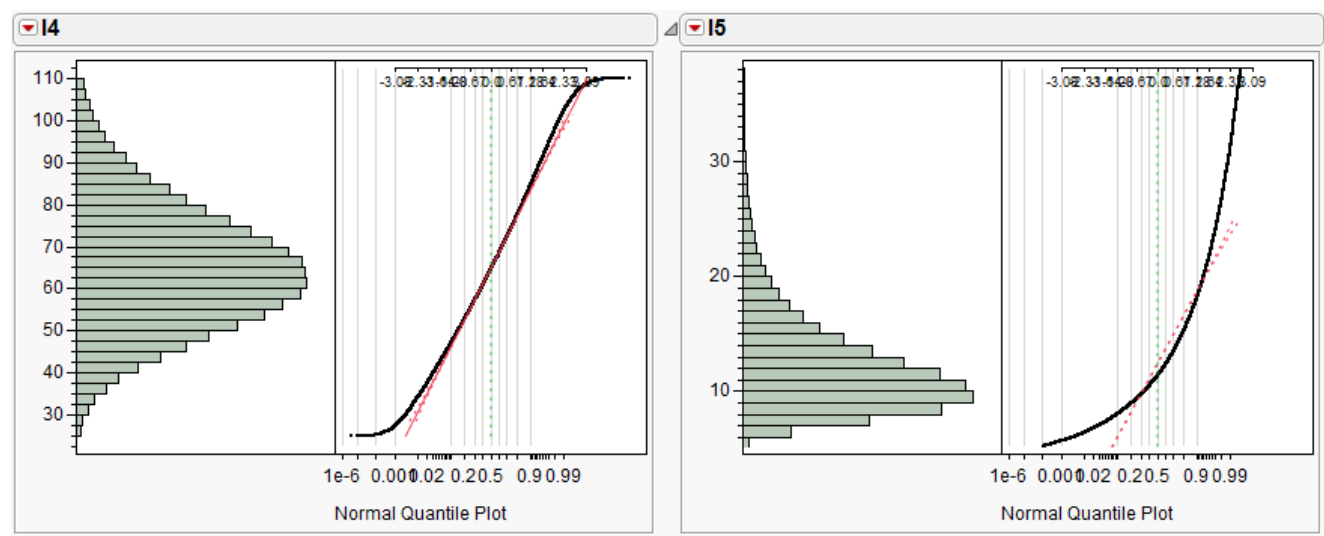

Fig. 4 The distribution of the input variables

\subsubsection{Homogeneity of variances and covariances}

The DA suggested that the linear discriminant analysis is appropriate when the variances and covariances of the two groups are the same. If the two groups have unequal variances and covariances, the 
quadratic discriminant analysis should be used instead [6]. Based on the collected data, the test of variances shown in Fig. 5 showed that the variances of the two groups were not equal. Therefore, the quadratic DA should be the appropriate tool.

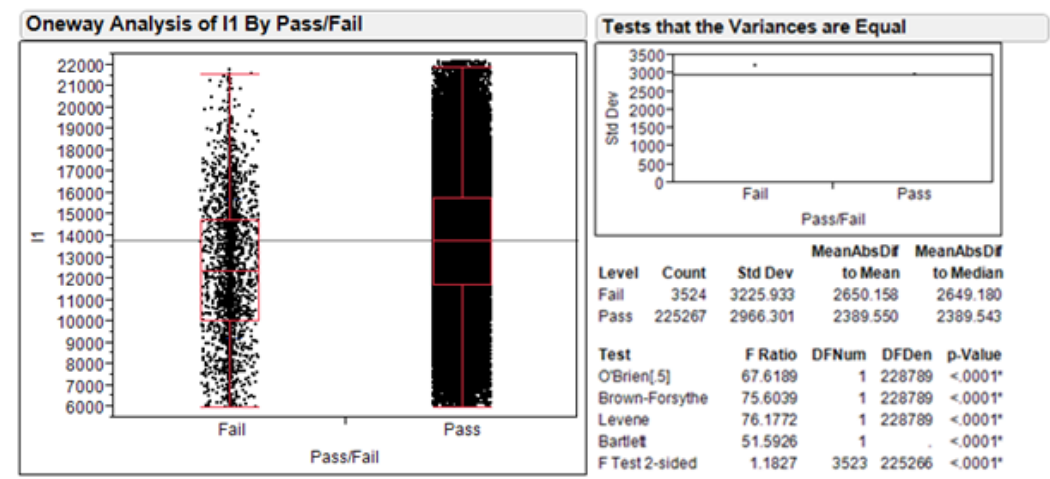

Fig. 5 Result of the test of variances

\subsubsection{Non-multicollinearity}

If one of the independent variables is very highly correlated with another, or one is a function (e.g., the sum) of other independent variables, then the tolerance value for that variable will approach 0 and the matrix will not have a unique discriminant solution [4]. After checking the correlations between all parameters, it was found that only variables I3 and E2 had high correlation of more than 0.7. Then, the stepwise regression analysis should be performed further to decide whether either one of these two variables should be excluded from the discriminant function.

Table 3 Correlation Matrix of Predicting Variables

\begin{tabular}{|c|c|c|c|c|c|c|c|c|c|c|c|c|}
\hline \multicolumn{13}{|c|}{ Correlations } \\
\hline & A1 & T1 & I1 & 12 & 13 & 14 & 15 & 16 & 17 & E1 & E2 & E3 \\
\hline A1 & 1.0000 & 0.0938 & -0.0117 & 0.0296 & -0.0679 & 0.0418 & -0.0271 & -0.0135 & -0.0152 & 0.0196 & -0.0682 & -0.0185 \\
\hline T1 & 0.0938 & 1.0000 & -0.0209 & 0.0318 & -0.0900 & 0.0510 & 0.0042 & 0.0117 & -0.0117 & 0.0418 & -0.0909 & -0.1680 \\
\hline 11 & -0.0117 & -0.0209 & 1.0000 & -0.2861 & 0.3312 & 0.6971 & -0.3604 & -0.1863 & -0.2447 & -0.0018 & 0.3159 & -0.0062 \\
\hline 12 & 0.0296 & 0.0318 & -0.2861 & 1.0000 & -0.3493 & -0.0005 & 0.0504 & 0.0407 & 0.0960 & 0.0075 & -0.3366 & -0.0079 \\
\hline 13 & -0.0679 & -0.0900 & 0.3312 & -0.3493 & 1.0000 & -0.4230 & 0.1541 & 0.0329 & 0.0463 & -0.0203 & 0.9915 & 0.0520 \\
\hline 14 & 0.0418 & 0.0510 & 0.6971 & -0.0005 & -0.4230 & 1.0000 & -0.4524 & -0.2019 & -0.2647 & 0.0147 & -0.4308 & -0.0466 \\
\hline 15 & -0.0271 & 0.0042 & -0.3604 & 0.0504 & 0.1541 & -0.4524 & 1.0000 & 0.2666 & 0.1632 & 0.0030 & 0.1555 & -0.0072 \\
\hline 16 & -0.0135 & 0.0117 & -0.1863 & 0.0407 & 0.0329 & -0.2019 & 0.2666 & 1.0000 & 0.3744 & 0.0038 & 0.0325 & 0.0049 \\
\hline 17 & -0.0152 & -0.0117 & -0.2447 & 0.0960 & 0.0463 & -0.2647 & 0.1632 & 0.3744 & 1.0000 & 0.0135 & 0.0492 & -0.0020 \\
\hline E1 & 0.0196 & 0.0418 & -0.0018 & 0.0075 & -0.0203 & 0.0147 & 0.0030 & 0.0038 & 0.0135 & 1.0000 & -0.0149 & 0.0388 \\
\hline E2 & -0.0682 & -0.0909 & 0.3159 & -0.3366 & 0.9915 & -0.4308 & 0.1555 & 0.0325 & 0.0492 & -0.0149 & 1.0000 & 0.0562 \\
\hline E3 & -0.0185 & -0.1680 & -0.0062 & -0.0079 & 0.0520 & -0.0466 & -0.0072 & 0.0049 & -0.0020 & 0.0388 & 0.0562 & 1.0000 \\
\hline
\end{tabular}

The stepwise regression result in Fig. 6 showed that out of the total 12 variables, there were 10 variables that were significant or were useful in the discriminant function. It was interesting to note that variables I3 and E2 which were highly correlated were not dropped from the function. That means the adding of these two variables significantly affected the prediction result.

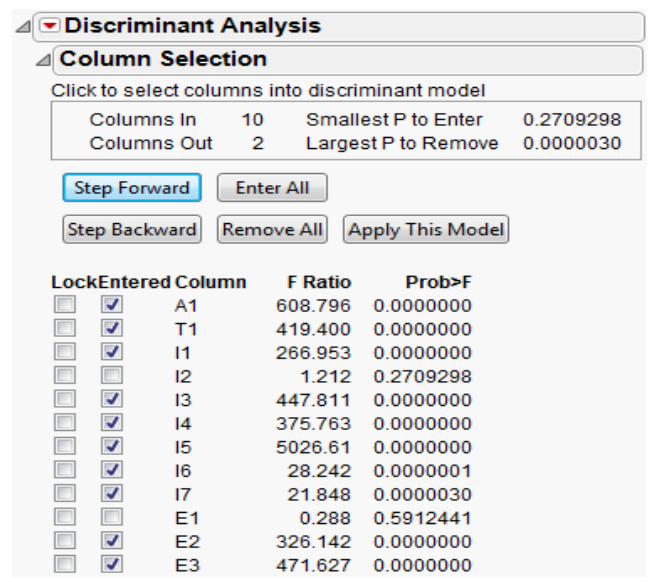

Fig. 6 Stepwise regression results of 12 predicting variables 


\subsection{Discriminant Function Creation}

The 10 significant variables were used to create the discriminant function. The Wilks' lambda test showed that the created discriminant function was significant or useful to use for prediction.

\subsection{Consideration on the Performance of the Prediction}

Table 4 Prediction Results Using the Discriminant Function
\begin{tabular}{|l|c|c|c|c|c|c|c|c|}
\hline & \multicolumn{3}{|c|}{ Analysis Sample } & \multicolumn{5}{c|}{ Validation Sample } \\
\cline { 2 - 11 } & Group 1 & Group 2 & Group 3 & Group 4 & Group 1 & Group 2 & Group 3 & Group 4 \\
\hline Prediction result & Pass & Pass & Fail & Fail & Pass & Pass & Fail & Fail \\
\hline Actual status & Pass & Fail & Pass & Fail & Pass & Fail & Pass & Fail \\
\hline Percentage of HGAs & 89.83 & 0.84 & 8.66 & 0.67 & 89.97 & 0.86 & 8.46 & 0.71 \\
\hline
\end{tabular}

It can be seen from Table 4 that the prediction in both Analysis Sample and Validation Sample were close. This result showed that the created discriminant function had sufficient repeatability. The percentage of the HGAs that were predicted as "pass" (group 1 and group 2) was $90.83 \%$. This number was the percentage of the HGAs that can skip the test at the HGA process.

Then, these results were transformed to show the performance of the predicting equation using four performance indicators as follows:

1) Percentage of correctly predicted HGAs (Group 1 and Group 4)

2) Percentage of misclassified HGAs

3) Percentage of fail HGAs that falsely accepted: $\beta$ (Group 2)

4) Percentage of pass HGAs that falsely rejected: $\alpha$ (Group 3)

Table 5 showed that the created discriminant function could predict the results with $90.68 \%$ accuracy. However, there were $0.86 \%$ of the fail HGAs that would be passed to the HDD process. These HGAs would adversely affect the yield of the HDD process.

Table 5 Performance of the Proposed Electrical Testing Plan (Sampling Test)

\begin{tabular}{|l|c|c|c|c|}
\cline { 2 - 5 } \multicolumn{1}{c|}{} & \% Correct & \% Misclassified & \% Beta & \% Alpha \\
\hline Analysis Sample & 90.50 & 9.50 & 0.84 & 8.66 \\
\hline Validation Sample & 90.68 & 9.32 & 0.86 & 8.46 \\
\hline
\end{tabular}

\subsection{Decision Making}

Since the percentage of correct prediction of $90.68 \%$ was higher than the criteria of $90 \%$ and the percentage of falsely accepting bad HGAs of $0.86 \%$ was less than the criteria of $1 \%$, thus the researcher decided that the created discriminant function or the proposed sampling test could be used instead of the current testing procedure. As a result, $90.83 \%$ of the HGAs could skip the test. This helped reduce the usage of the electrical testing machines of product A from 60 machines to only 48 machines or $20 \%$ reduction. This sampling test then helped reduce $10 \%$ of all testing machines usage.

\section{DISCUSSION}

This paper considers the performance of the proposed sampling test based on the performance of the prediction only. It is suggested that the effect of the proposed sampling test on the cost of quality such as the appraisal costs and the failure costs should be investigated further. In addition, the effect of the number of variables used in the prediction should be also further studied.

\section{CONCLUSION}

This research presents the sampling test as a method to reduce the testing activity, which helps reduce the usage of testing machines. This research proposes the new electronic sampling test of the Head Gimbal Assembly (HGA) that uses the discriminant analysis technique to predict whether each of the HGAs will pass or fail the on-disc test based on variables from previous testing processes. If the prediction results shows "pass", the company should skip the testing of that HGA but if the prediction result shows "fail", the company should test that HGA as usual.

Before making the prediction, the assumptions related to sample size, the distribution of variables, homogeneity of variances, and non-multicollinearity of variables have to be validated. Next, the significance of the discriminant function has to be proved in order to make sure that the created function will be suitable to 
make the prediction. Then, the performance of the prediction has to be evaluated and the user has to decide whether the performance is acceptable in order to use the discriminant function to make the prediction further. This case study showed that the discriminant function could predict the results with $90.68 \%$ accuracy. If applying this method in the production, the case study factory could reduce the testing machine usage up to $20 \%$ of product A or $10 \%$ of the testing machines in the factory. This left over capacity could be used for other products or to support increasing demand in the future without buying new testing machines.

\section{REFERENCES}

[1] N. Wongsin, J. Yunibhand, S. Suktakul, Discriminant analysis of relapse and non-relapse on Thai muslim schizophrenic patients, Chulalongkorn University, Bangkok, Thailand, 2012

[2] V. J. Harwood, J. Whitlock, V. Withington, Classification of antibiotic resistance patterns of indicator bacteria by discriminant analysis: use in predicting the source of fecal contamination in subtropical waters. Applied and Environmental Microbiology, 2000, 66(9), 3698-3704.

[3] H. Jo, I. Han, H. Lee, Bankruptcy prediction using case-based reasoning, neural networks, and discriminant analysis. Expert Systems with Applications, 1997, 13.2: 97-108.

[4] J. Poulsen, A. French, Discriminant function analysis, San Francisco, CA, 2004

[5] B.G Tabachnick, L.S. Fidell, Using multivariate statistics. New York, NY, 1996

[6] Ş. Büyüköztürk, Ö. Çokluk-Bökeoğlu, Discriminant function analysis: concept and application. Eurasian Journal of Educational Research, 2008, 33, 73-92. 